\title{
A Cosmological Model and Modifications to Einstein's Theory of Relativity
}

\author{
Jiancheng Liu \\ Dongfang Boiler Co., Ltd., Zigong, China \\ Email: decljc@163.com
}

Received 26 January 2016; accepted 10 February 2016; published 15 February 2016

Copyright (C) 2016 by author and OALib.

This work is licensed under the Creative Commons Attribution International License (CC BY).

http://creativecommons.org/licenses/by/4.0/

(c) (i) Open Access

\section{Abstract}

This paper studied the revisions to Einstein's theory of relativity, introduced the concept of "size mass" and put forward the new relativity mass-velocity formula; and then it put forward Liu's Cosmological Model, analyzed the nascence, birth and development of the universe, obtained the Universe Moving Equations, and calculated the present universe's parameters; and it analyzed the conditions that a celestial body occurs the similar big bang and the model that the Galaxy occurred the second big bang. The results show that at its nascence, the universe was a big neutron of which the temperature was $8.876 \times 10^{12}{ }^{\circ} \mathrm{C}$, the radius was $r_{0}=4.84 \times 10^{11} \mathrm{~m}$ and that was at rest, under the action of temperature energy (heat energy) releasing, the center of this big neutron generated repulsive force to the surrounding. Under the common action of the repulsive force and gravitation, at the moment $t_{b}=6.77 \times 10^{-5} \mathrm{~s}$, the Big Bang occurred. Since the Big Bang, most celestial bodies have been leaving the center of the universe and spreading around with velocities above $0.47 c$, and the universe is constantly expanding. When an object moves relative to the absolute frame of reference, its three-dimensional size will shrink by the same proportion. The fourdimensional Minkowski space-time is meaningless. The size mass of a system is reduced, if its temperature is maintained unchangeable, then its energy will be correspondingly reduced, and the other system accepts an increase of size mass, if its temperature is maintained unchangeable, then its energy is correspondingly increased.

\section{Keywords}

Einstein's Theory of Relativity, Mass-Velocity Formula, Liu's Cosmological Model, The Big Bang of Universe and the Universe Moving Equations

Subject Areas: Special Theory of Relativity, Theoretical Physics 


\section{Amendment to Einstein's Special Theory of Relativity}

\subsection{Frame of Reference}

We take the absolutely rest ether as the basic frame of reference, call the values measured in this frame of reference as absolute values or true values, and call this frame of reference as absolute frame of reference; while call the coordinate system which is moving with a constant velocity relative to the absolutely rest ether as the inertial frame, and call the values measured in this frame as relative values or observed values. The parameter without a prime is the absolute value of this parameter while the object is at absolutely rest, and the parameter with a prime is the relative value of this parameter while the object is moving relative to the absolutely rest ether. The measurement unit is the earth system unit (i.e. the international metric system), and the source to be traced is the present earth.

\subsection{Lorentz Transformation}

Suppose that the inertial frame is moving with a velocity $v$ (which is parallel to the axes $x$ and $x^{\prime}$ ) relative to the absolute frame of reference, we introduce the Lorentz Transformation ([1] [2]):

$$
\begin{aligned}
& x^{\prime}=\frac{x-v t}{\sqrt{1-\frac{v^{2}}{c^{2}}}} \\
& t^{\prime}=\frac{t-\frac{v}{c^{2}} \cdot x}{\sqrt{1-\frac{v^{2}}{c^{2}}}} .
\end{aligned}
$$

From Formulas (1) and (2), we get

$$
\begin{gathered}
x_{1}^{\prime}=\frac{x_{1}-v t_{1}}{\sqrt{1-\frac{v^{2}}{c^{2}}}} \\
x_{2}^{\prime}=\frac{x_{2}-v t_{2}}{\sqrt{1-\frac{v^{2}}{c^{2}}}} \\
t_{1}^{\prime}=\frac{t_{1}-\frac{v}{c^{2}} \cdot x_{1}}{\sqrt{1-\frac{v^{2}}{c^{2}}}} \\
t_{2}^{\prime}=\frac{t_{2}-\frac{v}{c^{2}} \cdot x_{2}}{\sqrt{1-\frac{v^{2}}{c^{2}}}} .
\end{gathered}
$$

So

$$
\begin{gathered}
\Delta x^{\prime}=x_{2}^{\prime}-x_{1}^{\prime}=\frac{1}{\sqrt{1-\frac{v^{2}}{c^{2}}}}\left[\left(x_{2}-x_{1}\right)-v\left(t_{2}-t_{1}\right)\right]=\frac{1}{\sqrt{1-\frac{v^{2}}{c^{2}}}}(\Delta x-v \cdot \Delta t) \\
\Delta t^{\prime}=t_{2}^{\prime}-t_{1}^{\prime}=\frac{1}{\sqrt{1-\frac{v^{2}}{c^{2}}}}\left[\left(t_{2}-t_{1}\right)-\frac{v}{c^{2}}\left(x_{2}-x_{1}\right)\right]=\frac{1}{\sqrt{1-\frac{v^{2}}{c^{2}}}}\left(\Delta t-\frac{v}{c^{2}} \cdot \Delta x\right) .
\end{gathered}
$$


For an object which is moving with a velocity $v$ together with the inertial frame, if we want to measure the length of this object, we must observe the two ends of it simultaneously, then $\Delta t^{\prime}=0$, inserting it into Formula (3) and (4), we get:

$$
\Delta x^{\prime}=\Delta x \cdot \sqrt{1-\frac{v^{2}}{c^{2}}}
$$

That's say, for an object moving with the velocity $v$, its length is contracted to be the $\sqrt{1-\frac{v^{2}}{c^{2}}}$ times of its length at absolute rest, i.e. its length become shorter.

The Formula (5) is the famous scale reduction (Lorentz contraction) formula.

For a point on this moving object, there are $\Delta x=0$ and $\Delta x^{\prime}=0$, inserting them into Formulas (3) and (4), we get $\Delta t^{\prime}=\Delta t=0$, while we can not get the time dilation (slow clock) formula $\Delta t^{\prime}=\frac{1}{\sqrt{1-\frac{v^{2}}{c^{2}}}} \Delta t$.

\subsection{The Error of Slow Clock Formula}

Suppose that there is a vacuum tube the length of which is $\Delta x$, when this vacuum tube is at absolutely rest, it needs a time of $\Delta t$ for the light to transmit a distance of $\Delta x$ in the tube, i.e. there is $c=\frac{\Delta x}{\Delta t}$. While if this vacuum tube is parallel to the axis $x^{\prime}$ of the relative frame along the direction of its length and moves together with the relative frame with a velocity $v$ (which is parallel to the axis $x^{\prime}$ ), the length of it will contract to be $\Delta x^{\prime}=\Delta x \cdot \sqrt{1-\frac{v^{2}}{c^{2}}}$. If the slow clock formula is tenable, i.e. it needs a time of $\Delta t^{\prime}=\frac{1}{\sqrt{1-\frac{v^{2}}{c^{2}}}} \Delta t$ for the light to transmit a distance of $\Delta x^{\prime}$ in the vacuum tube of the relative frame, and then there is:

$$
\frac{\Delta x^{\prime}}{\Delta t^{\prime}}=\left(1-\frac{v^{2}}{c^{2}}\right) \frac{\Delta x}{\Delta t}=\left(1-\frac{v^{2}}{c^{2}}\right) c \text {. }
$$

That's say, the transmission velocity of the light in the vacuum of the inertial frame is $\left(1-\frac{v^{2}}{c^{2}}\right) c$, the hypothesis that the velocity of light in vacuum is unchangeable is not tenable anymore. So the slow clock formula is wrong.

\subsection{The Error of the Derivation of the Time Dilation Formula of Einstein's Special Theory of Relativity}

Øyvind Grøn and Sigbjørn Hervik derived the time dilation formula $\Delta t=\frac{1}{\sqrt{1-\frac{v^{2}}{c^{2}}}} \Delta t^{\prime}$ of Einstein's Special Theory of Relativity by using the light clock model consisting of two parallel mirrors ([3]). Here, $\Delta t^{\prime}$ is the cycle of the light clock in a stationary frame of reference, and $\Delta t$ is the cycle of the light clock in a moving frame of reference, which is just the opposite of the stipulation of this article.

In fact, when the light clock moves with the velocity $v$ relative to the stationary frame of reference, supposing that the cycle of the light clock measured in this moving frame of reference is $\Delta t^{\prime}$, then the cycle of the light clock measured in the stationary frame of reference is: $\Delta t=\frac{1}{\sqrt{1-\frac{v^{2}}{c^{2}}}} \Delta t^{\prime}$. Then there is: $\Delta t^{\prime}=\Delta t \cdot \sqrt{1-\frac{v^{2}}{c^{2}}}$, that 
is to say, when light clock moves relative to the stationary frame of reference, the time that the light transmitted between the two mirrors is shortened. As the assumption that the speed of light is constant, the distance between the two mirrors has been shortened, from $L_{0}$ reduced to $L_{0}^{\prime}$, and there is $L_{0}^{\prime}=L_{0} \cdot \sqrt{1-\frac{v^{2}}{c^{2}}}$. This conclusion is consistent with the scale reduction formula. Here $\Delta t$ and $\Delta t^{\prime}$ both are a time interval, rather than the natural time. The natural time will not expand or shrink whether the frame of reference is moving or not, and it has nothing to do with the frame of reference, and is an independent natural variable. The natural time is one-way, always forward, never back. From the above analysis, it also shows that when an object moves relative to the absolute frame of reference, its three-dimensional size will shrink by the same proportion, that is $L_{0}^{\prime}=L_{0} \cdot \sqrt{1-\frac{v^{2}}{c^{2}}}$.

\subsection{The Error of Four-Dimensional Minkowski Space-Time}

A continuous four-dimensional space-time domain consisting of the whole set of $\{c t, x, y, z\}$ is the Minkowski space-time in the category of Einstein's special theory of relativity ([1] [2]). In fact, the value of the variable $c t$ is distance that the light transmits in the time interval $t$, while $\{x, y, z\}$ are the three-dimensional coordinates of an object which is stationary or moving with a velocity $v$. Because the light moves with the speed of light $c$, while the object moves with the velocity $v$, so the frame of reference for the light which moves with the speed of light $c$ and the frame of reference for the object which moves with the velocity $v$ do not overlap at all, and it is impossible to couple the both as a space-time, unless $v=c$. Therefore, the four-dimensional Minkowski spacetime is meaningless.

\section{The Size Mass and Gravitational Mass}

According to its original meaning, the "mass" should mean how much matter (i.e. the number of matter) a substance system of objective reality contains. The mass-velocity relation formula of Einstein's Theory of Relativi-

ty $m=\frac{m_{0}}{\sqrt{1-\frac{v^{2}}{c^{2}}}}$ should mean that: when an object is moving with a certain velocity relative to the absolute system, its inertia (gravitational mass) will increase, but it does not mean that the number of matter also increases. Hereon, we introduce the concept of "Size Mass", which means the number of matter that a substance system of objective reality contains and is independent of the moving velocity of the substance system, i.e. regardless the substance system is moving or not or moving with how high a velocity, its size mass is unchangeable. In fact, in any inertial system, the standard $\mathrm{kg}$ of the present earth system and the mass of a substance system measured on the present earth will be enlarged or lessen the same times, so the value of the mass of a substance system measured in the inertial system when the substance system is moving together with the inertial system is the same as the value of the mass of the substance system measured in the earth system when it is moving together with the earth; in the absolute system, the standard $\mathrm{kg}$ of the present earth system and the mass of a substance system measured on the present earth will be lessen the same times, so the value of the mass of a substance system measured in the absolute system when the substance system is at absolutely rest is the same as the value of the mass of the substance system measured in the earth system when it is moving together with the earth. Therefore, the size mass of a substance system is unchangeable. So called gravitational mass, it is the mass of an object that appears in the universal gravitation law formula. When a substance system is at absolutely rest, its gravitational mass is equal to its size mass. If it is not specially illuminated, the masses stated in this paper all are gravitational masses.

\section{Modifications to Einstein's Mass-Velocity Formula}

Introducing into Einstein’s mass-velocity formula:

$$
m=\frac{m_{0}}{\sqrt{1-\frac{v^{2}}{c^{2}}}}
$$


Formula (6) can be transformed as

$$
(m c)^{2}=\left(m_{0} c\right)^{2}+(m v)^{2} .
$$

The rest mass, moving mass and the velocity of light and velocity form a pythagorean theorem (Figure 1 ).

Nevertheless, Formula (7) and Figure 1 are only applicable to the condition that the mass energy $E=m c^{2}$ and the gravitational mass $m=\frac{m_{0}}{\sqrt{1-\frac{v^{2}}{c^{2}}}}$ of a object increase when a external force is doing work on the object at rest. While if no external force does work on a object at rest and the object releases energy, the mass energy $E=m c^{2}$ and the gravitational mass $m$ (its expression Temporarily unknown) will decrease, and the object will obtain a certain velocity $v$. the mass-velocity pythagorean theorem will change to be the expression (8) and Figure 2.

$$
\begin{gathered}
\left(m_{0} c\right)^{2}=(m c)^{2}+(m v)^{2} \\
m=\frac{m_{0}}{\sqrt{1+\frac{v^{2}}{c^{2}}}}
\end{gathered}
$$

Formula (9) is a new mass-velocity formula of relativity, called the second mass-velocity formula.

\section{Liu's Cosmological Model}

\subsection{The Mass of Universe}

The universe in which we live has about 200 billions of galaxies, the small galaxy has several billions of fixed stars, and the big galaxy has about 400 billions of fixed stars, so averagely each galaxy has about 200 billions of fixed stars. The Sun in the solar system in which we stay is a middle size fixed star, and the mass of the Sun is $1.99 \times 10^{30} \mathrm{~kg}$. Hereby, we can get that the masses of the visible matters in the universe are $7.57 \times 10^{52} \mathrm{~kg}$. The astronomical observations and the scientific researches discovered that the absolutely most matters in the universe are invisible dark matters, and the mass of visible matters such as fixed stars etc. is only $5 \%$ of the universe matters. So, the size mass of all the matters of the universe in which we live is:

$$
M_{U S}=1.51 \times 10^{54} \mathrm{~kg} \text {. }
$$

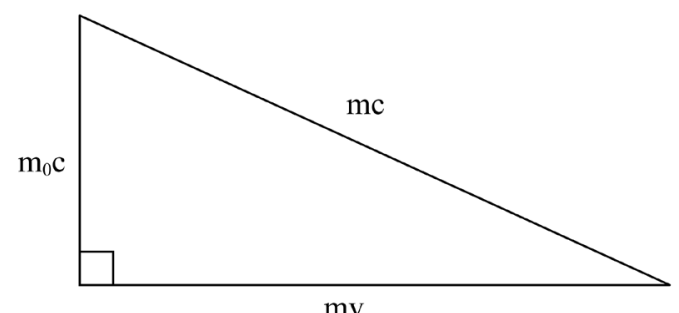

mv

Figure 1. Mass-velocity pythagorean theorem I.

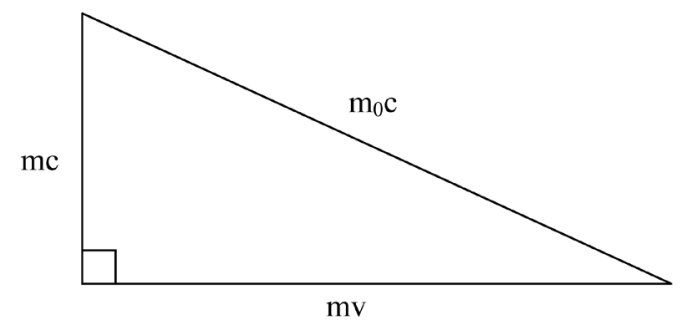

Figure 2. Mass-velocity pythagorean theorem II. 


\subsection{The Nascence of Universe and Big Bang}

\subsubsection{The Nascence of Universe}

The diameter of a neutron measured on the earth of the solar system is about $r_{n}=5 \times 10^{-16} \mathrm{~m}$, and the rest mass (also the size mass) of a neutron measured in the earth system is:

$$
m_{n}=1.008665 u=1.008665 \times 1.66 \times 10^{-27} \mathrm{~kg} \approx 1.67 \times 10^{-27} \mathrm{~kg} .
$$

At this time, the density of the neutron is:

$$
\rho_{n}=\frac{m_{n}}{\frac{4}{3} \pi r_{n}^{3}}=\frac{1.67 \times 10^{-27}}{\frac{4}{3} \times 3.142 \times\left(5 \times 10^{-16}\right)^{3}}=3.19 \times 10^{18}\left(\mathrm{~kg} / \mathrm{m}^{3}\right) .
$$

At its nascence, the universe was a big neutron at rest and with sky-high temperature, supposed that this big neutron had the same density, and then we can get its radius as:

$$
r_{0}=\left(\frac{M_{U 0}}{m_{n}}\right)^{\frac{1}{3}} r_{n}=\left(\frac{1.51 \times 10^{54}}{1.67 \times 10^{-27}}\right)^{\frac{1}{3}} \times\left(5 \times 10^{-16}\right)=4.84 \times 10^{11}(\mathrm{~m}) \gg 1 \mathrm{~m} .
$$

\subsubsection{The Big Bang of Universe}

It can be deemed that the energy of any material composes of three parts, i.e. the mass energy, the temperature energy (heat energy) and the moving energy (kinetic energy). Supposed that size mass of this big neutron was $M_{U 0}$, density was $\rho_{0}$, temperature was $T_{0}$ and specific heat was $C_{U O}$, then at its nascence, the mass energy of the universe was $M_{U 0} c^{2}$, the temperature energy (heat energy) was $M_{U 0} C_{U 0} T_{0}$, the kinetic energy was 0 , and the total energy was $E_{U 0}=M_{U 0} c^{2}+M_{U 0} C_{U 0} T_{0}$. While at the moment of the big bang, the size mass of the universe measured was also $M_{U 0}$, the gravitational mass is $M_{U b}$, the moving velocity was $c$, the average temperature is $T_{b}$, the average specific heat was $C_{U b}$, then the total energy of the universe at the big bang was $E_{U b}=M_{U b} c^{2}+M_{U 0} C_{U b} T_{b}+\frac{1}{2} M_{U b} c^{2}$. According to the law of conservation of energy, there is:

$$
E_{U 0}=M_{U 0} c^{2}+M_{U 0} C_{U 0} T_{0}=M_{U b} c^{2}+M_{U 0} C_{U b} T_{b}+\frac{1}{2} M_{U b} c^{2} .
$$

Under the action of temperature energy (heat energy) releasing, the center of this big neutron generated repulsive force to the surrounding. While under the common action of the repulsive force and gravitation, at first the repulsive force greater than the gravitation, the surrounding of this big neutron began to expand at accelerating speed. Supposed that at the moment of the natural time $t=t_{b}$ and the radius of this big neutron was $r_{b}$, the repulsive force balanced with the gravitation, the moving velocities of the particles at the surrounding reached at $c$, and the Big Bang occurred. Hereinafter, we analyze the movement process of a particle at the edge of this big neutron before and after the big bang.

Supposed that the size mass of this particle is $\mathrm{dm}_{0}$, the gravitational mass in the process of movement is $\mathrm{dm}$, its distance to the center of the universe is $r$, and the moving velocity is $v$ (departure from the center of the universe is positive, pointing to the center of the universe is negative), then there is:

$$
r=r_{0}+\int_{0}^{t} v \mathrm{~d} t
$$

Set the expressions of the repulsive force and the gravitation are respectively:

$$
\begin{gathered}
F_{R}=R \frac{M_{U 0} \mathrm{~d} m}{r^{3}} \\
F_{G}=-G \frac{M_{U 0} \mathrm{~d} m}{r^{2}} .
\end{gathered}
$$

At the moment of big bang, $t=t_{b}, \quad r=r_{b}, \quad F=F_{R}+F_{G}=0$, so: $R=r_{b} G$. 
The acceleration of this particle is:

$$
a=R \frac{M_{U 0}}{r^{3}}-G \frac{M_{U 0}}{r^{2}}=G \frac{M_{U 0}}{r^{2}}\left(\frac{r_{b}}{r}-1\right) \geq 0
$$

i.e.

$$
\frac{\mathrm{d} v}{\mathrm{~d} t}=G M_{U 0}\left(\frac{1}{r_{0}+\int_{0}^{t} v \mathrm{~d} t}\right)^{2}\left(\frac{r_{b}}{r_{0}+\int_{0}^{t} v \mathrm{~d} t}-1\right) \geq 0 .
$$

$G$ is the gravitational constant, $G=6.673 \times 10^{-11} \mathrm{~N} \cdot \mathrm{m}^{2} / \mathrm{kg}^{2}$.

$$
a_{0}=G \frac{M_{U 0}}{r_{0}^{2}}\left(\frac{r_{b}}{r_{0}}-1\right)
$$

When $t=t_{b} \ll 1 \mathrm{~s}, a_{t_{b}}=0$, then between the time 0 and $t_{b}$, the average value of $a$ is: $\bar{a}=\frac{1}{2} a_{0}$.

$$
r_{b}=r_{0}+\frac{1}{2} \overline{a t}_{b}^{2}=r_{0}+\frac{1}{4} a_{0} t_{b}^{2}
$$

Thus we can get:

$$
t_{b}=\sqrt{\frac{4 r_{0}^{3}}{G M_{U O}}}
$$

Considering that $M_{U O}=\frac{4}{3} \pi r_{0}^{3} \rho_{0}$, so

$$
t_{b}=\sqrt{\frac{4 r_{0}^{3}}{G M_{U O}}}=\sqrt{\frac{3}{\pi G \rho_{0}}} .
$$

While at $t=t_{b}, \quad v=\bar{a} t_{b}=\frac{1}{2} a_{0} t_{b}=c$, so

$$
\begin{gathered}
a_{0}=\frac{2 c}{t_{b}}=\frac{2 \times 3 \times 10^{8}}{6.77 \times 10^{-5}}=8.86 \times 10^{12} \mathrm{~m} / \mathrm{s} \\
r_{b}=r_{0}+\frac{1}{4} a_{0} t_{b}^{2}=4.84 \times 10^{11}+\frac{1}{4} \times 8.86 \times 10^{12} \times\left(6.77 \times 10^{-5}\right)^{2} \\
=\left(4.84 \times 10^{11}+1.02 \times 10^{4}\right) \mathrm{m}
\end{gathered}
$$

We take a similar analysis for the internal particles of this big neutron as follows.

As for $r<r_{0}, a=R \frac{M}{r^{3}}-G \frac{M}{r^{2}}=G \frac{M}{r^{2}}\left(\frac{r_{b}}{r}-1\right)$.

while $M=\left(\frac{r}{r_{0}}\right)^{3} M_{U 0}$

$$
a=G \frac{M_{U 0}}{r_{0}^{2}} \frac{r}{r_{0}}\left(\frac{r_{b}}{r}-1\right)
$$

and $r_{b}=r+\frac{1}{2} \bar{a} t_{b}^{2}=r+\frac{1}{4} a t_{b}^{2}$, similarly we can get

$$
t_{b}=\sqrt{\frac{4 r_{0}^{3}}{G M_{U O}}}=\sqrt{\frac{3}{\pi G \rho_{0}}} .
$$


Inserting the related data into Formula (12), we get:

$$
t_{b}=\sqrt{\frac{4 r_{0}^{3}}{G M_{U O}}}=\sqrt{\frac{4 \times\left(4.84 \times 10^{11}\right)^{3}}{6.673 \times 10^{-11} \times 1.51 \times 10^{54}}}=6.77 \times 10^{-5} \mathrm{~s} .
$$

\subsubsection{The Related Parameters of the Big Bang}

As the big bang occurred under the condition that no external force was doing work to this big neutron, it released heat energy, and its mass energy and gravitational mass was decreasing, so the second mass-velocity formula is tenable, there is

$$
M_{U b}=\frac{M_{U 0}}{\sqrt{1+\frac{c^{2}}{c^{2}}}}=\frac{\sqrt{2}}{2} M_{U 0} .
$$

Inserting into Formula (10), get

$$
M_{U 0} c^{2}+M_{U 0} C_{U 0} T_{0}=\frac{\sqrt{2}}{2} M_{U 0} c^{2}+M_{U 0} C_{U b} T_{b}+\frac{\sqrt{2}}{4} M_{U 0} c^{2} .
$$

Supposed that after the big bang, the temperature of the universe decreased sharply, and compared with $M_{U 0} C_{U 0} T_{0}, \quad M_{U 0} C_{U b} T_{b}$ can be ignored, then there is

$$
T_{0}=\left(\frac{3 \sqrt{2}}{4}-1\right) \frac{c^{2}}{C_{U 0}} .
$$

Of all matters known at present, the specific heat of water is the largest and is $4.2 \times 10^{3} \mathrm{~J} /(\mathrm{kg} \cdot \mathrm{K})$, it can be supposed might as well that at the nascence of the universe, the specific heat of this big neutron also was $4.2 \times 10^{3} \mathrm{~J} /(\mathrm{kg} \cdot \mathrm{K})$, then

$$
T_{0}=\left(\frac{3 \sqrt{2}}{4}-1\right) \frac{\left(3 \times 10^{8}\right)^{2}}{4.2 \times 10^{3}}=1.28 \times 10^{12} \mathrm{~K} .
$$

So, at its nascence, the universe was a big neutron of which the temperature was $8.876 \times 10^{12}{ }^{\circ} \mathrm{C}$, the radius was $r_{0}=4.84 \times 10^{11} \mathrm{~m}$ and that was at rest, under the action of temperature energy (heat energy) releasing, the center of this big neutron generated repulsive force to the surrounding. Under the common action of the repulsive force and gravitation, at the moment $t_{b}=6.77 \times 10^{-5} \mathrm{~s}$, the Big Bang occurred.

After the big bang occurred, the main forms that the matters existed were the electrons, photons and neutrinos. Afterwards, the matters were continuously expanding and spreading because their velocities were slowing down, the temperature rapidly decreased, and the chemical elements began to take shape. As the temperature was decreasing continuously, atoms were taking shape continuously. At the same time, as the temperature was decreasing, the temperature energy was changing into mass energy continuously, and the gravitational mass of the universe was increasing continuously. The universe was filled with the gas clouds, under the effect of gravity they are forming star systems, and after another long evolution, the stellar systems became today's universe.

Today's universe is composed of galaxies, all galaxies are leaving from the center of the universe and diffusing outwards; a galaxy is composed of a number of stars and all stars are rotating around the center of the galaxy; and each star has a number of planets rotating around it. In addition, a planet may have a number of satellites rotating around it.

\subsection{The Universe Moving Equations after Big Bang}

After the big bang, the most of the universal materials was spreading at the velocity of light $c$. Supposed that during the first year after the big bang, the velocities of these materials basically maintained at $c=3 \times 10^{8} \mathrm{~m} / \mathrm{s}$, then, when $t>t_{1}=1 \mathrm{yr}=3.15 \times 10^{7} \mathrm{~s}, r>r_{1}=r_{b}+\int_{t_{b}}^{t} v \mathrm{~d} t=9.45 \times 10^{15} \mathrm{~m}$, the most of the universe mass is con- 
centrated outside the sphere of $r=9.45 \times 10^{15} \mathrm{~m}$, and the mass of the universe center decreased rapidly to $M_{1}$. As the gravitation was bigger than the repulsive force, the velocities of the particles at this sphere begun to decrease, and their acceleration were:

$$
a=-\left(G \frac{M_{1}}{r^{2}}-R \frac{M_{1}}{r^{3}}\right)=-G \frac{M_{1}}{r^{2}}\left(1-\frac{r_{b}}{r}\right) .
$$

Then

$$
\frac{\mathrm{d} v}{\mathrm{~d} t}=-G M_{1}\left(\frac{1}{r_{1}+\int_{t_{1}}^{t} v \mathrm{~d} t}\right)^{2}\left(1-\frac{r_{b}}{r_{1}+\int_{t_{1}}^{t} v \mathrm{~d} t}\right)
$$

As $1-\frac{r_{b}}{r_{1}+\int_{t_{1}}^{t} v \mathrm{~d} t} \approx 1-\frac{r_{b}}{r_{1}}=1-5.12 \times 10^{-5} \approx 1$, Formula (13) is simplified as:

$$
\frac{\mathrm{d} v}{\mathrm{~d} t}=-G M_{1}\left(\frac{1}{r_{1}+\int_{t_{1}}^{t} v \mathrm{~d} t}\right)^{2}
$$

Solving Equation (14), we get:

$$
\frac{\mathrm{d} v}{\mathrm{~d} t}=-\frac{1}{4}\left(\frac{v^{2}}{\sqrt{G M_{1}}}+C_{1}\right)^{2}
$$

Inserting the boundary conditions $t=t_{1}=3.15 \times 10^{7} \mathrm{~s}, \quad v=c=3 \times 10^{8}(\mathrm{~m} / \mathrm{s})$ and $\frac{\mathrm{d} v}{\mathrm{~d} t}=\left(\frac{\mathrm{d} v}{\mathrm{~d} t}\right)_{t_{1}}=-\frac{G M_{1}}{r_{1}^{2}}$ into Equation (15), we get:

$$
C_{1}=\frac{2 \sqrt{G M_{1}}}{r_{1}}-\frac{c^{2}}{\sqrt{G M_{1}}} .
$$

According to the physical meaning of Equation (15), there must be:

$$
C_{1}=\frac{2 \sqrt{G M_{1}}}{r_{1}}-\frac{c^{2}}{\sqrt{G M_{1}}}<0 \text {. }
$$

Set

$$
b=\frac{c^{2}}{\sqrt{G M_{1}}}-\frac{2 \sqrt{G M_{1}}}{r_{1}}>0
$$

Then there is:

$$
M_{1}<\frac{r_{1} c^{2}}{2 G}=\frac{9.45 \times 10^{15} \times\left(3 \times 10^{8}\right)^{2}}{2 \times 6.673 \times 10^{-11}}=6.37 \times 10^{42} \mathrm{~kg} .
$$

If $M_{1} \geq \frac{r_{1} c^{2}}{2 G}=6.37 \times 10^{42} \mathrm{~kg}$, the universe center would be a black hole, after the big bang of the universe, even a matter which was moving with the velocity of light would be attracted by the excessively strong gravitation of the universe center and could not spread outwards, and the universe would not expand to the present size.

So, there is

$$
\frac{\mathrm{d} v}{\mathrm{~d} t}=-\frac{1}{4}\left[\frac{v^{2}}{\sqrt{G M_{1}}}-b\right]^{2}
$$


Hereinafter, we solve Equation (16).

From Equation (16), we get:

$$
\frac{\mathrm{d} v^{2}}{\mathrm{~d} t}=-\frac{1}{2} v\left[\frac{v^{2}}{\sqrt{G M_{1}}}-b\right]^{2} .
$$

Set $u=\frac{v^{2}}{\sqrt{G M_{1}}}-b$, then

$$
v=\left(G M_{1}\right)^{\frac{1}{4}} \sqrt{u+b} .
$$

Then there is:

$$
\frac{\mathrm{d} u}{\mathrm{~d} t}=-\frac{1}{2}\left(G M_{1}\right)^{-\frac{1}{4}} u^{2} \sqrt{u+b} .
$$

i.e.:

$$
\frac{\mathrm{d} u}{u^{2} \sqrt{u+b}}=-\frac{1}{2}\left(G M_{1}\right)^{-\frac{1}{4}} \mathrm{~d} t .
$$

More, set $\alpha=\sqrt{u+b}$, then $u=\alpha^{2}-b, \mathrm{~d} u=2 \alpha \mathrm{d} \alpha$, and there is:

$$
\int_{u_{1}}^{u} \frac{\mathrm{d} u}{u^{2} \sqrt{u+b}}=\frac{2}{b^{2}} \int_{\alpha_{1}}^{\alpha} \frac{\mathrm{d} \alpha}{\left[\left(\frac{\alpha}{\sqrt{b}}\right)^{2}-1\right]^{2}} .
$$

Of which, $u_{1}=\frac{c^{2}}{\sqrt{G M_{1}}}-b, \alpha_{1}=\sqrt{u_{1}+b}=\left(G M_{1}\right)^{-\frac{1}{4}} C$.

And more, set $\sec \beta=\frac{\alpha}{\sqrt{b}}$, then $\alpha=\sqrt{b} \sec \beta, \mathrm{d} \alpha=\sqrt{b} \sec \beta \tan \beta \mathrm{d} \beta$, Equation (17) change to be:

$$
\begin{aligned}
& \frac{2}{b^{2}} \int_{\alpha_{1}}^{\alpha} \frac{\mathrm{d} \alpha}{\left[\left(\frac{\alpha}{\sqrt{b}}\right)^{2}-1\right]^{2}}=2 b^{-\frac{3}{2}} \int_{\beta_{1}}^{\beta}\left(\frac{1}{\sin ^{3} \beta}-\frac{1}{\sin \beta}\right) \mathrm{d} \beta \\
& =2 b^{-\frac{3}{2}}\left[\left(\frac{\cos \beta}{-2 \sin ^{2} \beta}+\frac{\cos \beta_{1}}{2 \sin ^{2} \beta_{1}}\right)-\frac{1}{2} \int_{\beta_{1}}^{\beta} \frac{\mathrm{d} \beta}{\sin \beta}\right] \\
& =b^{-\frac{3}{2}}\left[\left(\frac{\cos \beta_{1}}{\sin ^{2} \beta_{1}}-\frac{\cos \beta}{\sin ^{2} \beta}\right)+\left(\ln \left|\tan \frac{\beta_{1}}{2}\right|-\ln \left|\tan \frac{\beta}{2}\right|\right)\right]
\end{aligned}
$$

of which $\beta_{1}=\sec ^{-1}\left(\frac{\alpha_{1}}{\sqrt{b}}\right)=\sec ^{-1}\left(\frac{c}{\left(G M_{1}\right)^{\frac{1}{4}} \sqrt{b}}\right)$.

While $\int_{t_{1}}^{t}-\frac{1}{2}\left(G M_{1}\right)^{-\frac{1}{4}} \mathrm{~d} t=-\frac{1}{2}\left(G M_{1}\right)^{-\frac{1}{4}}\left(t-t_{1}\right)$.

So, from the above analysis, we get:

$$
\begin{gathered}
\left(\frac{\cos \beta_{1}}{\sin ^{2} \beta_{1}}-\frac{\cos \beta}{\sin ^{2} \beta}\right)+\left(\ln \left|\tan \frac{\beta_{1}}{2}\right|-\ln \left|\tan \frac{\beta}{2}\right|\right)=-\frac{1}{2} b^{\frac{3}{2}}\left(G M_{1}\right)^{-\frac{1}{4}}\left(t-t_{1}\right) \\
v=\left(G M_{1}\right)^{\frac{1}{4}} \sqrt{b} \sec \beta .
\end{gathered}
$$


We call equations (19) and (20) as the Universe Moving Equations.

Of which, $G=6.673 \times 10^{-11} \mathrm{~N} \cdot \mathrm{m}^{2} / \mathrm{kg}^{2}$

$$
\beta_{1}=\sec ^{-1}\left(\frac{\alpha_{1}}{\sqrt{b}}\right)=\sec ^{-1}\left(\frac{c}{\left(G M_{1}\right)^{\frac{1}{4}} \sqrt{b}}\right) .
$$

Take $M_{1}=5 \times 10^{42} \mathrm{~kg}$, then

$$
\begin{gathered}
b=\frac{c^{2}}{\sqrt{G M_{1}}}-\frac{2 \sqrt{G M_{1}}}{r_{1}}=\frac{\left(3 \times 10^{8}\right)^{2}}{\sqrt{6.673 \times 10^{-11} \times 5 \times 10^{42}}}-\frac{2 \times \sqrt{6.673 \times 10^{-11} \times 5 \times 10^{42}}}{9.45 \times 10^{15}}=1.1 \\
\beta_{1}=\sec ^{-1}\left(\frac{c}{\left(G M_{1}\right)^{\frac{1}{4}} \sqrt{b}}\right)=\sec ^{-1}\left(\frac{3 \times 10^{8}}{\left(6.673 \times 10^{-11} \times 5 \times 10^{42}\right)^{\frac{1}{4}} \sqrt{1.1}}\right)=0.344 \pi .
\end{gathered}
$$

Inserting the above data into equations (19) and (20), there are:

$$
\begin{gathered}
\left(0.6-\frac{\cos \beta}{\sin ^{2} \beta}\right)+\left(0.63-\ln \left|\tan \frac{\beta}{2}\right|\right)=-4.26 \times 10^{-9}\left(t-3.15 \times 10^{7}\right) \text { or } \\
1.23-\frac{\cos \beta}{\sin ^{2} \beta}-\ln \left|\tan \frac{\beta}{2}\right|=-4.26 \times 10^{-9}\left(t-3.15 \times 10^{7}\right) \\
v=0.47 c \sec \beta .
\end{gathered}
$$

To verify Equation (22), insert $\beta=\beta_{1}=0.344 \pi$, we get:

$$
v=0.47 c \sec (0.344 \pi)=0.999 c \text {. }
$$

The Formula (22) is tenable.

Equations (21) and (22) namely are the perfect Universe Moving Equations.

\subsection{The Parameters of the Present Universe}

The Big Bang has taken place about 15 billion years $\left(4.73 \times 10^{17}\right.$ seconds). Hereinafter, we seek the expanding speed and the radius of the present universe.

Inserting $t=t_{150}=4.73 \times 10^{17} \mathrm{~s}$ into Formula (21), there is

$$
1.23-\frac{\cos \beta}{\sin ^{2} \beta}-\ln \left|\tan \frac{\beta}{2}\right|=-4.26 \times 10^{-9} \times\left(4.73 \times 10^{17}-3.15 \times 10^{7}\right)=-2.0 \times 10^{9} \text {. }
$$

We get:

$$
\begin{gathered}
\beta_{150}=2.24 \times 10^{-5} \\
v_{150}=0.47 c \sec \left(2.24 \times 10^{-5}\right)=0.47 c .
\end{gathered}
$$

When the time $t=\infty, \beta_{\infty}=0, v_{\infty}=0.47 \mathrm{c}$.

So, the scope of the values of $\beta$ is between $0.344 \pi$ and 0 , and the scope of the values of $v$ is between $c$ and $0.47 c$, most celestial bodies have been leaving the center of the universe and spreading around with velocities above $0.47 c$, and the universe is constantly expanding.

The radius of present universe is:

$$
r_{150}=r_{1}+\frac{c+0.47 c}{2} \times 150 \times 10^{8} \mathrm{ly} \approx 110 \times 10^{8} \mathrm{ly}
$$

i.e., 11 billions light-years. 


\subsection{The Conditions That a Similar Big Bang of a Celestial Body Occurs}

For a celestial body with big mass, sky-high temperature and low velocity, suppose that its size mass is $M_{C}$, density is $\rho_{C}$, radius is $r_{C}$, temperature is $T_{C}$ and specific heat is $C_{C}$, then its mass energy is $M_{C} c^{2}$, the temperature energy (heat energy) is $M_{C} C_{C} T_{C}$, the kinetic energy is neglectable and the total energy is $E_{C}=\sqrt{2} M_{C} c^{2}+M_{C} C_{C} T_{C}$, and the time the big bang of this celestial body is $t_{C b}$, then there is:

$$
t_{C b}=\sqrt{\frac{4 r_{C}^{3}}{G M_{C}}}=\sqrt{\frac{3}{\pi G \rho_{C}}} \text { and } r_{C} \gg 1 \text {. }
$$

Only if the conditions $t_{C b}=\sqrt{\frac{4 r_{C}^{3}}{G M_{C}}}=\sqrt{\frac{3}{\pi G \rho_{C}}} \ll 1 \mathrm{~s}$ and $r_{C} \gg 1 \mathrm{~m}$ are satisfied, a similar big bang of the celestial body maybe will occur.

\section{The Secondary Explosion of the Galaxy}

\subsection{The Model for the Secondary Explosion of the Galaxy}

The size mass of the Galaxy is $1 \times 10^{12}$ times of the size mass of the Sun, i.e. $1.99 \times 10^{42} \mathrm{~kg}$; the size mass of the Galaxy center at present is $3.7 \times 10^{6}$ times of the size mass of the Sun, i.e. $7.36 \times 10^{36} \mathrm{~kg}$. Supposed that 10 billions years after the birth of the universe, i.e. 5 billions years ago, the secondary explosion of the Galaxy occurred, and at this time the Galaxy center was a big neutron star with sky-high temperature. Inset $t=t_{100}=3.15 \times 10^{17} \mathrm{~s}$ into Equations (21) and (22), it can be calculated out: $v_{100}=0.47 \mathrm{csec}\left(2.74 \times 10^{-5}\right)=0.47 \mathrm{c}$.

Supposed that this big neutron star had the same density, and then we can get its radius as:

$$
r_{G}=\left(\frac{M_{G}}{m_{n}}\right)^{\frac{1}{3}} r_{n}=\left(\frac{1.99 \times 10^{42}}{1.67 \times 10^{-27}}\right)^{\frac{1}{3}} \times\left(5 \times 10^{-16}\right)=5.30 \times 10^{7} \mathrm{~m} \gg 1 \mathrm{~m} .
$$

Inserting into Formula (23), we get:

$$
t_{G b}=\sqrt{\frac{4 r_{G}^{3}}{G M_{G}}}=\sqrt{\frac{4 \times\left(5.30 \times 10^{7}\right)^{3}}{6.673 \times 10^{-11} \times 1.99 \times 10^{42}}}=6.70 \times 10^{-5} \mathrm{~s} \ll 1 \mathrm{~s} .
$$

So, the original neutron star of the Galaxy had the condition to occur the secondary explosion.

Through an analysis similar to paragraph 4.2, and ignoring the effects of the universe center and the celestial bodies, we can get the moving equations of the Galaxy celestial bodies:

$$
\begin{gathered}
\left(\frac{\cos \beta_{G 1}}{\sin ^{2} \beta_{G 1}}-\frac{\cos \beta}{\sin ^{2} \beta}\right)+\left(\ln \left|\tan \frac{\beta_{1}}{2}\right|-\ln \left|\tan \frac{\beta}{2}\right|\right)=-\frac{1}{2} b^{\frac{3}{2}}\left(G M_{G 1}\right)^{-\frac{1}{4}}\left(t-t_{G 1}\right) \\
v=\left(G M_{G 1}\right)^{\frac{1}{4}} \sqrt{b} \sec \beta .
\end{gathered}
$$

Of which, if take $t_{G 1}=100 \mathrm{~s}, \quad r_{G 1}=r_{G b}+\int_{t_{G b}}^{t} v \mathrm{~d} t=3 \times 10^{10} \mathrm{~m}$.

So, $M_{G 1}<\frac{r_{G 1} c^{2}}{2 G}=\frac{3 \times 10^{10} \times\left(3 \times 10^{8}\right)^{2}}{2 \times 6.673 \times 10^{-11}}=2.02 \times 10^{37} \mathrm{~kg}$.

Take $M_{G 1}=7.36 \times 10^{36} \mathrm{~kg}$, then

$$
b=\frac{c^{2}}{\sqrt{G M_{G 1}}}-\frac{2 \sqrt{G M_{G 1}}}{r_{G 1}}=\frac{\left(3 \times 10^{8}\right)^{2}}{\sqrt{6.673 \times 10^{-11} \times 7.36 \times 10^{36}}}-\frac{2 \times \sqrt{6.673 \times 10^{-11} \times 7.36 \times 10^{36}}}{3 \times 10^{10}}=2590
$$




$$
\beta_{G 1}=\sec ^{-1}\left(\frac{c}{\left(G M_{G 1}\right)^{\frac{1}{4}} \sqrt{b}}\right)=\sec ^{-1}\left(\frac{3 \times 10^{8}}{\left(6.673 \times 10^{-11} \times 7.36 \times 10^{36}\right)^{\frac{1}{4}} \sqrt{2590}}\right) \approx 0.206 \pi .
$$

Inserting the above data into equations (24) and (25), we get:

$$
\begin{gathered}
\left(2.19-\frac{\cos \beta}{\sin ^{2} \beta}\right)+\left(-1.09-\ln \left|\tan \frac{\beta}{2}\right|\right)=-1.4 \times 10^{-5}(t-100) \text { or } \\
1.1-\frac{\cos \beta}{\sin ^{2} \beta}-\ln \left|\tan \frac{\beta}{2}\right|=-1.4 \times 10^{-5}(t-100) \\
v=0.8 c \sec \beta .
\end{gathered}
$$

Equations (26) and (27) namely are the perfect Moving Equations of the Galaxy celestial bodies.

\subsection{The Parameters of the Galaxy Celestial Bodies Moving}

The secondary explosion of the galaxy has taken place for about 5 billions years $\left(1.58 \times 10^{17} \mathrm{~s}\right)$. Hereinafter, we seek the expanding speed and the radius of the present Galaxy.

Inserting $t=t_{50}=1.58 \times 10^{17} \mathrm{~s}$ into Formula (26), there is

$$
1.1-\frac{\cos \beta}{\sin ^{2} \beta}-\ln \left|\tan \frac{\beta}{2}\right|=-1.4 \times 10^{-5} \times\left(1.58 \times 10^{17}-100\right)=-2.21 \times 10^{12} \text {. }
$$

We get:

$$
\begin{gathered}
\beta_{50}=6.7 \times 10^{-7} \\
v_{50}=0.8 c \sec \left(6.7 \times 10^{-7}\right) \approx 0.8 c .
\end{gathered}
$$

Most celestial bodies in the Galaxy have been leaving the center of the Galaxy and spreading around with velocities above $0.80 c$.

The radius of present Galaxy is:

$$
r_{50}=r_{G 1}+\frac{c+0.8 c}{2} \times 50 \times 10^{8} \mathrm{ly} \approx 45 \times 10^{8} \mathrm{ly}
$$

i.e., 4.5 billions light-years.

\subsection{Will the Sun Have a Big Bang?}

The current cosmological theory deems that: the sun was originally one and one hydrogen atom just produced by a secondary explosion of the Galaxy, due to the effect of gravity, which accumulated in together, becoming a sphere, and the sun was born.

The size mass of the Sun is $1.99 \times 10^{30} \mathrm{~kg}$ and its diameter is $1.39 \times 10^{9} \mathrm{~m}$. Inserting them into Formula (23), we can get:

$$
t_{C b}=\sqrt{\frac{4 r_{C}^{3}}{G M_{C}}}=\sqrt{\frac{4 \times\left(6.95 \times 10^{8}\right)^{3}}{6.673 \times 10^{-11} \times 1.99 \times 10^{30}}}=3.18 \times 10^{3} \mathrm{~s} \gg 1 \mathrm{~s} .
$$

So the Sun could not have a big bang.

\subsection{Black Holes}

Most celestial bodies are moving with velocities above $0.47 c$, only if their radii $r$ and gravitational masses $M$ satisfy the relationship formula $r<\frac{2 G M}{c^{2}}$, they could not shine and the lights that arrive their vicinity will be 
completely absorbed, and these kinds of celestial bodies namely are black holes.

\section{The Summary of Liu's Cosmological Model}

At its nascence, the universe was a big neutron of which the temperature was $8.876 \times 10^{12 \circ} \mathrm{C}$, the radius was $r_{0}=4.84 \times 10^{11} \mathrm{~m}$ and that was at rest, under the action of temperature energy (heat energy) releasing, the center of this big neutron generated repulsive force to the surrounding. Under the common action of the repulsive force and gravitation, at the moment $t_{b}=6.77 \times 10^{-5} \mathrm{~s}$, the Big Bang occurred. After the big bang occurred, the main forms that the matters existed were the electrons, photons and neutrinos. Afterwards, the matters were continuously expanding and spreading because their velocities were slowing down, the temperature rapidly decreased, and the chemical elements began to take shape. As the temperature was decreasing continuously, atoms were taking shape continuously. At the same time, as the temperature was decreasing, the temperature energy was changing into mass energy continuously, and the gravitational mass of the universe was increasing continuously. The universe was filled with the gas clouds, under the effect of gravity they are forming star systems, and after another long evolution, the stellar systems became today's universe.

Today's universe is composed of galaxies, all galaxies are leaving from the center of the universe and diffusing outwards; a galaxy is composed of a number of stars and all stars are rotating around the center of the galaxy; and each star has a number of planets rotating around it. In addition, a planet may have a number of satellites rotating around it. So the structure of the universe is quite stable. Since the Big Bang, most celestial bodies have been leaving the center of the universe and spreading around with velocities above $0.47 c$, and the universe is constantly expanding. Only if the conditions $t_{C b}=\sqrt{\frac{4 r_{C}^{3}}{G M_{C}}}=\sqrt{\frac{3}{\pi G \rho_{C}}} \ll 1 \mathrm{~s}$ and $r_{C} \gg 1 \mathrm{~m}$ are satisfied, a similar big bang of the celestial body maybe will occur. The original neutron star of the Galaxy had the condition to occur the secondary explosion; most celestial bodies in the Galaxy have been leaving the center of the Galaxy and spreading around with velocities above $0.80 c$. The Sun was originally one and one hydrogen atom just produced by a secondary explosion of the Galaxy, due to the effect of gravity, which accumulated in together, becoming a sphere, and the sun was born. The Sun could not have a big bang. Most celestial bodies are moving with velocities above $0.47 c$, only if their radii $r$ and gravitational masses $M$ satisfy the relationship formula $r<\frac{2 G M}{c^{2}}$, they could not shine and the lights that arrive their vicinity will be completely absorbed, and these kinds of celestial bodies namely are black holes.

\section{The Rest Mass of Photon}

According to Plank's Quantum Theory, the energy of a photon which is moving is $E_{p}=h v$. As the photon is produced from a matter which releases energy, so the gravitational mass of the photon can not satisfy the Einstein's mass-velocity formula, but satisfy the second mss-velocity formula, i.e.:

$$
m=\frac{m_{0}}{\sqrt{1+\frac{c^{2}}{c^{2}}}}=\frac{\sqrt{2}}{2} m_{0}
$$

$m_{0}$ is the rest mass of a photon.

According to Equation (10), the energy of photon is:

$$
E_{p}=\frac{\sqrt{2}}{2} m_{0} c^{2}+m_{0} C_{p} T_{p}+\frac{\sqrt{2}}{4} m_{0} c^{2} .
$$

Ignoring the temperature energy (heat energy), there is:

$$
\begin{gathered}
E_{p}=\frac{\sqrt{2}}{2} m_{0} c^{2}+\frac{\sqrt{2}}{4} m_{0} c^{2}=\frac{3 \sqrt{2}}{4} m_{0} c^{2} . \\
m_{0}=\frac{2 \sqrt{2}}{3} \frac{h v}{c^{2}}
\end{gathered}
$$


Plank's Constant $h=6.63 \times 10^{-34} \mathrm{~J} \cdot \mathrm{s}$.

As for visible light, the scope of its wavelength is $0.4-0.76 \mu \mathrm{m}$, the velocity of light is $c=3 \times 10^{8} \mathrm{~m} / \mathrm{s}$, then its frequency scope is $v=(3.95-7.50) \times 10^{14} \mathrm{~Hz}$. According to Formula (28), it can be calculated out the absolutely rest masses (size masses) of the photon of visible lights are $m_{0}=(2.74-5.21) \times 10^{-36} \mathrm{~kg}$.

An experiment accomplished by Chinese scientists shows that, in any conditions, the mass of a photon cannot exceed $10^{-54} \mathrm{~kg}$, this result is the $1 / 20$ of the upper limit of the photon's mass known before.

Reported by Scientific Times, this innovational achievement was published in authoritative Physical Review Letters. Heretofore, most physicists deem that the particles that transmit the light-photons have no masses. At the same time, they wish this point of view can be proved by experiments.

The scientists said: this is like a match the amount of stake for which is huge. The classic electromagnetism theory forbids that the photon has any mass. If this theory is no longer applicable, then consequences it will bring about will be radical-for example, the velocity of light will change as its wavelength changes, and the light wave will have longitudinal vibration like a sound wave.

This experiment related to the mass of photon is accomplished by Professor Luo Jun together with his colleagues of Huazhong University of Science and Technology, Wuhan, Hubei, PRC. Supported by the National Natural Science Fund of China, they conducted the study "to verify the upper limit of the rest mass of photon using precision torsion balance”, and through the experiment to look for the trace of photon mass in the effect caused by the universal magneto motive force which is brought about by the magnetic field of a galaxy as well as galaxy group. According to the sensitivity of this experiment they conducted, it is deduced that the mass of photon must be less than $10^{-48} \mathrm{~kg}$ or the $1 / 10^{24}$ of an electron's mass.

It can be seen that, the conclusion of the above experiment has some deviation, the rest masses of visible light are $m_{0}=(2.74-5.21) \times 10^{-36} \mathrm{~kg}$.

\section{The Moving Law of an Object in Solar System}

\subsection{Low Speed Moving Law}

The synthesized velocity formula of the Einstein's Theory of Relativity is as follows:

$$
u=\frac{v_{1}+v_{2}}{1+\frac{v_{1} v_{2}}{c^{2}}}
$$

where, $v_{1}=$ the moving velocity of the inertial frame;

$v_{2}=$ the speed of an object moving relative to the inertial frame;

$u=$ the absolute moving velocity of the object;

$c=$ the universal utmost velocity.

In the solar system, $v_{1}=v_{S}=0.8 c$, suppose that the moving speed of an object in the solar system (i.e. relative to the solar system) has a general expression $v_{2}=a \times 10^{-n} c$ (in the same direction as the Sun moving) or $v_{2}=-a \times 10^{-n} c$ (in the opposite direction as the Sun moving)(as for a larger object, the moving speed of it commonly is less than the third astronomical speed, i.e. $\left|v_{2}\right|<16.7 \mathrm{~km} / \mathrm{s}=5.57 \times 10^{-5} \mathrm{c}$, then there are:

a) $v_{2}$ in the same direction as the Sun moving

$$
u=\frac{0.8+a \times 10^{-n}}{1+0.8 \times\left(a \times 10^{-n}\right)} c=\left[0.8+0.36 a \times 10^{-n}\right] c .
$$

b) $v_{2}$ in the opposite direction as the Sun moving

$$
u=\frac{\left(0.8-a \times 10^{-n}\right)}{1-0.8 \times a \times 10^{-n}} c=\left[0.8-0.36 a \times 10^{-n}\right] c .
$$

Suppose that the mass of the object when is at relative rest in the solar system (i.e. $v_{2}=0$ ) is $M_{0}$, then, when it is moving relative to the solar system with the speed $v_{2}$, its maximum mass $M_{\max }$ has the expression 
as follows:

$$
M_{\text {max }}=\frac{\sqrt{1-0.8^{2}}}{\sqrt{1-\left[0.8+0.36 a \times 10^{-n}\right]^{2}}} M_{0} \approx\left(1+0.8 \times a \times 10^{-n}\right) M_{0} .
$$

While the expression of its minimum mass $M_{\min }$ is:

$$
M_{\min }=\frac{\sqrt{1-0.8^{2}}}{\sqrt{1-\left[0.8-0.36 a \times 10^{-n}\right]^{2}}} M_{0} \approx\left(1-0.8 a \times 10^{-n}\right) M_{0} .
$$

When $v_{2}=16.7 \mathrm{~km} / \mathrm{s}=5.57 \times 10^{-5} \mathrm{c}$,

$$
\begin{aligned}
& M_{\text {max }}=\left(1+4.46 \times 10^{-5}\right) M_{0} \\
& M_{\text {min }}=\left(1-4.46 \times 10^{-5}\right) M_{0} .
\end{aligned}
$$

That's say when an object is moving with the third astronomical speed in the solar system, its maximum moving mass is $44.6 \mathrm{ppm}$ larger than its relative rest mass, and its minimum moving mass is $44.6 \mathrm{ppm}$ less than its relative rest mass. These two values (the same) are relatively very small, generally could be ignored. So, even in an inertial frame which is moving with a velocity near to the velocity of light $c$ (e.g. the solar system), the Newton Mechanics can still work with an object which is moving (relative to the inertial frame) with a low speed.

\subsection{High Speed Moving Law}

Take the electron circling the nuclei with a high speed as an example: suppose the speed of the electron moving relative to the solar system has a general expression $v_{e}=0.9 c$ (in the same direction as the Sun moving) or $v_{e}=-0.9 c$ (in the opposite direction as the Sun moving), then there are:

a) $v_{e}$ in the same direction as the Sun moving

$$
u=\frac{0.9+0.8}{1+0.9 \times 0.8} c=\left(1-1.2 \times 10^{-2}\right) c .
$$

b) $v_{e}$ in the opposite direction as the Sun moving

$$
u=\frac{0.9-0.8}{1-0.9 \times 0.8} c=0.357 c \text {. }
$$

Suppose that the mass of the electron when is at relative rest in the solar system (i.e. $v_{e}=0$ ) is $M_{0}$, then, when it is moving relative to the solar system with the speed $v_{e}$, its maximum mass $M_{\max }$ has the expression as follows:

$$
M_{\max }=\frac{\sqrt{1-0.8^{2}}}{\sqrt{1-\left(1-1.2 \times 10^{-2}\right)^{2}}} M_{0}=3.88 M_{0} .
$$

While the expression of its minimum mass $M_{\min }$ is:

$$
M_{\min }=\frac{\sqrt{1-0.8^{2}}}{\sqrt{1-0.357^{2}}} M_{0}=0.64 M_{0} .
$$

Thus it can be seen, for an electron circling the nuclei with a high speed, its mass is varying from the minimum $0.64 M_{0}$ to the maximum $3.88 M_{0}$. So, the orbits that the electron circles the nucleus are elliptical orbits and the nucleus is at one of these elliptical focuses. The electron in the hydrogen nuclei can not be an exception, and its moving orbit is not a round orbit. At the same time, because the direction of the Galaxy expanding is fixed, i.e. from the center of the Galaxy points to the center of the Sun, and the earth is moving relative to the center of the Galaxy and its moving direction is varying, so, the electron's moving orbits measured at different 
times and different places are also varying, i.e., its orbits are not repeated, so an electronic cloud is created.

\section{Evaluation of Einstein's General Theory of Relativity}

According to the foregoing analysis, the premise that Einstein's general theory of relativity can be tenableEinstein's special theory of relativity has serious mistakes, therefore, the many contents of Einstein's general theory of relativity are wrong and shall be reconstructed on the basis of Liu's theory of relativity.

The mass-energy equation shows that mass and energy are inseparable and connected. Mass or energy is each other's carrier, and if there is no mass, then there is no energy; if there is no energy, there is no mass. So, all things which have energies always have masses, the light and radio waves are no exception; the absolute vacuum has no mass, so it impossibly has energy. The quantity of any matter system can either be measured by its mass $m$ or measured by its energy $E$. The energy of a system is reduced, if its temperature is maintained unchangeable, then the size mass of it will be correspondingly reduced, and the other system accepts an increase of energy, if its temperature is maintained unchangeable, then its size mass will be correspondingly increased; the size mass of a system is reduced, if its temperature is maintained unchangeable, then its energy will be correspondingly reduced, and the other system accepts an increase of size mass, if its temperature is maintained unchangeable, then its energy is correspondingly increased.

\section{The Questions Left for the Followers to Resolve}

January 25, 2016, the Liu's Theory of Relativity was born. Like the everything of universe, it will continuously develop and grow forever. Up to now, the questions about the gestation, birth and growth of the universe are basically resolved; the Liu's Theory of Relativity strides the first step. From now on, it is the continual followers who may push the theory go forwards forever.

On the day of birth of Liu's Theory of Relativity, I leave several questions for the followers:

(1) How the life was of nascence?

(2) How the Solar system will continue to develop?

(3) How the human being and the society will continue to develop?

\section{Conclusions}

The slow clock formula is wrong, and the natural time will not expand or shrink whether the frame of reference is moving or not, and it has nothing to do with the frame of reference, and is an independent natural variable. The natural time is one-way, always forward, never back. When an object moves relative to the absolute frame of reference, its three-dimensional size will shrink by the same proportion. The four-dimensional Minkowski space-time is meaningless.

According to its original meaning, the "mass" should mean how much matter (i.e. the number of matter) a substance system of objective reality contains. The mass-velocity relation formula of Einstein's Theory of Relativity should mean that: when an object is moving with a certain velocity relative to the absolute system, its inertia (gravitational mass) will increase, but it does not mean that the number of matter also increases. "Size Mass" means the number of matter a substance system of objective reality contains and is independent of the moving velocity of the substance system, i.e. regardless the substance system is moving or not or moving with how high a velocity, its size mass is unchangeable.

At its nascence, the universe was a big neutron of which the temperature was $8.876 \times 10^{12}{ }^{\circ} \mathrm{C}$, the radius was $r_{0}=4.84 \times 10^{11} \mathrm{~m}$ and that was at rest, under the action of temperature energy (heat energy) releasing, the center of this big neutron generated repulsive force to the surrounding. Under the common action of the repulsive force and gravitation, at the moment $t_{b}=6.77 \times 10^{-5} \mathrm{~s}$, the Big Bang occurred. After the big bang occurred, the main forms that the matters existed were the electrons, photons and neutrinos. Afterwards, the matters were continuously expanding and spreading because their velocities were slowing down, the temperature rapidly decreased, and the chemical elements began to take shape. As the temperature was decreasing continuously, atoms were taking shape continuously. At the same time, as the temperature was decreasing, the temperature energy was changing into mass energy continuously, and the gravitational mass of the universe was increasing continuously. The universe was filled with the gas clouds, under the effect of gravity they are forming star systems, and after another long evolution, the stellar systems became today's universe. 
Today's universe is composed of galaxies; all galaxies are leaving from the center of the universe and diffusing outwards; a galaxy is composed of a number of stars and all stars are rotating around the center of the galaxy; and each star has a number of planets rotating around it. In addition, a planet may have a number of satellites rotating around it. So the structure of the universe is quite stable. Since the Big Bang, most celestial bodies have been leaving the center of the universe and spreading around with velocities above $0.47 c$, and the universe is constantly expanding. Only if the conditions $t_{C b}=\sqrt{\frac{4 r_{C}^{3}}{G M_{C}}}=\sqrt{\frac{3}{\pi G \rho_{C}}} \ll 1 \mathrm{~s}$ and $r_{C} \gg 1 \mathrm{~m}$ are satisfied, a similar big bang of the celestial body maybe will occur. The original neutron star of the Galaxy had the condition to occur the secondary explosion; most celestial bodies in the Galaxy have been leaving the center of the Galaxy and spreading around with velocities above $0.80 c$. The Sun was originally one and one hydrogen atom just produced by a secondary explosion of the Galaxy, due to the effect of gravity, which accumulated in together, becoming a sphere, and the Sun was born. The Sun could not have a big bang. Most celestial bodies are moving with velocities above $0.47 c$, only if their radii $r$ and gravitational masses $M$ satisfy the relationship formula $r<\frac{2 G M}{c^{2}}$; they could not shine and the lights that arrive their vicinity will be completely absorbed, and these kinds of celestial bodies namely are black holes.

As the photon is produced from a matter which releases energy, so the gravitational mass of the photon can not satisfy the Einstein's mass-velocity formula, but satisfy the second mss-velocity formula $m=\frac{m_{0}}{\sqrt{1+\frac{v^{2}}{c^{2}}}}$. The absolutely rest masses (size masses) of the photon of visible lights are $m_{0}=(2.74-5.21) \times 10^{-36} \mathrm{~kg}$.

For an electron circling the nuclei with a high speed, its mass is varying from the minimum $0.64 M_{0}$ to the maximum $3.88 M_{0}$. So, the orbits that the electron circles the nucleus are elliptical orbits and the nucleus is at one of these elliptical focuses. The electron in the hydrogen nuclei can not be an exception, and its moving orbit is not a round orbit. At the same time, because the direction of the Galaxy expanding is fixed, i.e. from the center of the Galaxy points to the center of the Sun, and the earth is moving relative to the center of the Galaxy and its moving direction is varying, so, the electron's moving orbits measured at different times and different places are also varying, i.e., its orbits are not repeated, so an electronic cloud is created.

Because the premise that Einstein's general theory of relativity can be tenable-Einstein's special theory of relativity has serious mistakes, therefore, many contents of Einstein's general theory of relativity are wrong and shall be reconstructed on the basis of Liu's theory of relativity. The mass and energy are inseparable and connected. Mass or energy is each other's carrier, and if there is no mass, then there is no energy; if there is no energy, there is no mass. So, all things which have energies always have masses; the light and radio waves are no exception; the absolute vacuum has no mass, so it impossibly has energy. The energy of a system is reduced, if its temperature is maintained unchangeable, then the size mass of it will be correspondingly reduced, and the other system accepts an increase of energy; if its temperature is maintained unchangeable, then its size mass will be correspondingly increased; the size mass of a system is reduced; if its temperature is maintained unchangeable, then its energy will be correspondingly reduced; and the other system accepts an increase of size mass; if its temperature is maintained unchangeable, then its energy is correspondingly increased.

\section{References}

[1] Einstein, A. (2006) Relativity, Special and the General Theory (The Popular Exposition). Edition 1, Peking University Press, Bejing.

[2] Liu, L., Fei, B.-J. and Zhang, Y.-Z. (2008) Special Theory of Relativity. Edition 2, Science Press, Beijing.

[3] Grøn, Ø. and Hervik, S. (2007) Einstein’s General Theory of Relativity-With Modern Applications in Cosmology. Springer Science +Business Media, LLC. 
Appendix: The Solution of Equation $\frac{\mathrm{d} v}{\mathrm{~d} t}=-G M_{U 0}\left(\frac{1}{r_{b}+\int_{t_{b}}^{t} v \mathrm{~d} t}\right)^{2}$

From the original equation, we get:

$$
\sqrt{v^{\prime}}=\frac{\sqrt{G M_{U 0}}}{r_{b}+\int_{t_{b}}^{t} v \mathrm{~d} t} i
$$

That is

$$
r_{b}+\int_{t_{b}}^{t} v \mathrm{~d} t=\left(\sqrt{\frac{G M_{U 0}}{v^{\prime}}}\right) i .
$$

Seeking the derivations of the both sides of Equation (A-1), we get:

$$
v=-\frac{\sqrt{G M_{U 0}}}{2} \frac{v^{\prime \prime}}{\left(v^{\prime}\right)^{\frac{3}{2}}} i
$$

That is

$$
v^{\prime \prime}=\frac{2 i}{\sqrt{G M_{U 0}}} v\left(v^{\prime}\right)^{\frac{3}{2}} .
$$

Setting $v^{\prime}=P(t)$, then we get:

$$
v^{\prime \prime}=\frac{\mathrm{d} P}{\mathrm{~d} t}=\frac{\mathrm{d} P}{\mathrm{~d} v} \cdot \frac{\mathrm{d} v}{\mathrm{~d} t}=P \frac{\mathrm{d} P}{\mathrm{~d} v} .
$$

Inserting into Equation (A-3) and by trimming, we get:

$$
\frac{\mathrm{d} P}{\sqrt{P}}=\frac{2 i}{\sqrt{G M_{U 0}}} v \mathrm{~d} v .
$$

Integrating the both sides of Equation (A-4), there is:

$$
2 P^{\frac{1}{2}}=\left(\frac{v^{2}}{\sqrt{G M_{U 0}}}+C_{1}\right) i
$$

That is

$$
P=\frac{\mathrm{d} v}{\mathrm{~d} t}=-\frac{1}{4}\left(\frac{v^{2}}{\sqrt{G M_{U 0}}}+C_{1}\right)^{2}
$$

